\title{
MODELOS PARA ESTIMATIVA DA ÁREA FOLIAR DA MANGUEIRA UTILIZANDO MEDIDAS LINEARES ${ }^{1}$
}

\author{
RENATA TRINDADE DE LIMA², PAULO JORGE DE OLIVEIRA PONTE DE SOUZA², \\ JULIANA CHAGAS RODRIGUES ${ }^{4}$, MARCUS JOSÉ ALVES DE LIMA ${ }^{5}$
}

RESUMO - Objetivou-se determinar os modelos que melhor descrevem a relação entre as dimensões lineares e a área foliar da mangueira, cultivar Tommy Atkins, além de estabelecer o fator de correção. A pesquisa foi conduzida na Fazenda Experimental da Universidade Federal Rural da Amazônia, na vila de Cuiarana, município de Salinópolis. Foram analisadas de 90 a 150 folhas, em 11 árvores de um campo experimental, determinando-se o comprimento e a largura máxima das folhas. A área foliar real foi determinada usando o integrador de área foliar "Area Meter" (AM 300). O fator de correção foi obtido pela divisão da área foliar real pela área foliar calculada. A área foliar foi também estimada por meio de modelos linear e não linear. Os modelos gerados apresentaram ótimo desempenho, com valores de $\mathrm{R}^{2}$ entre 90 e $97 \%$. O fator de correção de 0,71 pode ser utilizado para estimar a área foliar real da mangueira, multiplicado pelas medidas lineares. Os modelos que utilizaram o produto das dimensões como variável independente apresentaram valores de $\mathrm{R}^{2}$ superiores aos que utilizaram apenas uma das dimensões, sendo o tipo cúbico o mais preciso.

Termos para indexação: Mangifera indica L., área real da folha, comprimento e largura, modelo linear, modelo não linear, método não destrutivo.

\section{MODELS FOR ESTIMATING LEAF AREA OF MANGO, USING LINEAR MEASURES}

\begin{abstract}
The objective was to determine the models that best describe the relationship between linear dimensions and leaf area of mango, cv. Tommy Atkins, and to establishing the correction factor. The research has been conducted at the experimental farm of Federal Rural University of Amazonia, at Cuiarana village, municipality of Salinópolis, Pará. 90-150 leaves from 11 trees were analyzed in a field experiment and it was determined the length and width of leaves. The leaf area was determined using the actual of foliar area integrator Area Meter (AM 300). The correction factor was obtained by dividing the real foliar area by foliar area calculated. Linear and nonlinear models also estimated leaf area. The mathematical models created present an excellent performance, with $\mathrm{R}^{2}$ values between 90 and $97 \%$. The correction factor of 0.71 can be used to estimate the involving real leaf area of mango, multiplied by linear measurements. The models that used the product dimensions, as independent variables, had $\mathrm{R}^{2}$ values higher than those who used only one dimension, being the cubic model, the most accurate.
\end{abstract}

Index terms: Mangifera indica L., real leaf area, length and width, linear model, non linear model, non destructive method.

\section{INTRODUÇÃO}

Aárea foliar (AF) é uma das mais importantes medidas de avaliação do crescimento vegetal. Com isso, sua estimativa é de suma importância, uma vez que a arquitetura da copa e os efeitos da interceptação da radiação solar pela folhagem interferem na produtividade e na composição dos frutos (MARACAJÁ et al., 2008). Além disso, o conhecimento da AF permite a estimativa da perda de água por transpiração, devido às folhas serem os principais órgãos responsáveis pelas trocas gasosas entre a planta e o ambiente, e pelo processo fotossintético que depende da absorção da energia luminosa e sua conversão em energia química (PEREIRA et al., 1997; FAVARIN et al., 2002).

O índice de área foliar (IAF) é um dos componentes utilizados para descrever a área foliar, conceituado como adimensional, que representa a relação entre o total de uma das faces do tecido fotossintético por unidade de área da superfície do solo (JONCKHEERE et al., 2004). Esse componente

\footnotetext{
1(Trabalho 140-12). Recebido em: 10-04-2012. Aceito para publicação em: 27-06-2012.

${ }^{2}$ MSc. em Agronomia, UFRA, Belém-PA. E-mail: renata.lima@ufra.edu.br.

${ }^{3}$ Dr. em Meteorologia Agrícola, Prof ${ }^{\circ}$. Adjunto do Instituto Sócio Ambiental e dos Recursos Hídricos, ISARH/UFRA, Belém-PA. E-mail: paulo.jorge@ufra.edu.br.

${ }^{4}$ Msc. em Agronomia, UFRA, Belém-PA. E-mail: juliana.rodrigues@ufra.edu.br

${ }^{5}$ Msc. em Agronomia, UFRA, Belém-PA. E-mail: marcus.lima@ufra.edu.br
} 
é chave de entrada em modelos de simulação do crescimento e desenvolvimento vegetal, de interceptação de luz, de produção de biomassa, consumo de água, fixação de $\mathrm{CO}_{2}$ e comparação entre espécies e cultivares (ASNER et al., 2003; SINOQUET et al., 2007; TAKEDA et al., 2008).

Aárea das folhas pode ser obtida por métodos indiretos não destrutivos, como os modelos ou equações matemáticas que apresentam boa precisão para estimar a área foliar real em função das dimensões lineares, como o comprimento, a largura ou ambos. Tal procedimento é interessante devido a sua facilidade e rapidez na execução e, principalmente, pelo baixo custo, uma vez que é independente de equipamentos modernos e caros, além da não destruição da planta, o que permite que a medida seja feita várias vezes no mesmo indivíduo (SILVA et al., 2002; ARAÚJO et al., 2005).

Diversos pesquisadores desenvolveram equações que relacionam o comprimento, a largura da folha, ou ambos, para a cultura do feijão-vagem (QUEIROGA et al., 2003), algodão (MONTEIRO et al., 2005), girassol (ROUPHAEL et al., 2007), feijã -caupi (LIMA et al., 2008), dentre outras culturas (MARACAJÁ et al., 2008; FAGUNDES et al., 2009), com alto grau de precisão.

A relação entre a área real e o produto do comprimento pela largura máxima gera o termo denominado "fator de correção", o qual estima a área real de qualquer outra folha da mesma espécie, ao ser multiplicado pelo produto de suas dimensões lineares (BARROS et al., 1973). Tyagi e Devi (1988) utilizaram o valor de fator de correção igual a 0,86 para a mangueira, tomando como base folhas de formato elipsoidais, não sendo, no entanto, específico para essa cultura, uma vez que a mesma apresenta folhas de formato lanceolado (CUNHA; CASTRO NETO, 2000; CUNHA et al., 2002). Prasada et al. (1994) e Araújo et al. (2005),em seus estudos sobre estimativa de área foliar da mangueira, usando o produto do comprimento pela largura máxima da folha, determinaram fatores de correção de $0,64 \mathrm{e}$ 0,74 , respectivamente.

No entanto, as pesquisas desenvolvidas sobre a estimativa da área foliar da mangueira ainda são muito raras, necessitando de mais estudos com a própria cultura em questão, com diferentes cultivares e diferentes locais de estudo. Uma vez que a área foliar pode ser uma importante ferramenta na avaliação do crescimento e da produtividade das plantas, o objetivo deste trabalho foi determinar os modelos que melhor descrevem a relação entre as dimensões lineares e a área da folha da mangueira, cultivar Tommy Atkins, além de estabelecer o fator de correção, possibilitando a estimativa da área foliar de forma não destrutiva.

\section{MATERIAL E MÉTODOS}

A pesquisa foi conduzida em um pomar de mangueiras cv. Tommy Atkins, na Fazenda Experimental da Universidade Federal Rural da Amazônia, localizada na Vila de Cuiarana, município de Salinópolis, Pará (0³9'49,72’'S; 47¹7’03,41'”O; 17m). O pomar foi plantado para fins comerciais em 1993, em fileiras orientadas no sentido leste-oeste, com espaçamento de 10x10 m, perfazendo uma área total de aproximadamente 25 hectares. Foram selecionadas, aleatoriamente, 11 plantas dentro de uma área de um hectare, previamente demarcada para estudo. Para cada planta, selecionada foram coletadas 90 folhas (para oito plantas) e 150 folhas (para três plantas) escolhidas entre os terços inferior, médio e superior de cada planta, de modo a considerar folhas de todos os tamanhos e larguras.

As folhas fotossinteticamente ativas e não danificadas foram separadas das demais partes da planta, na inserção entre o limbo e o pecíolo. Em cada folha, determinaram-se o comprimento (C) ao longo da nervura central e a largura máxima (L) como a maior dimensão perpendicular ao alinhamento da nervura central, ambos em centímetro, utilizando-se de régua de $50 \mathrm{~cm}$. Através das medidas lineares (comprimento e largura máxima), determinou-se, a partir do produto, a área do retângulo que circunscreve a folha (área foliar calculada) (BARROS et al., 1973).

A área foliar real foi determinada usando o integrador de área foliar portátil "Area Meter" (modelo AM 300, ADC BioScientific, Inglaterra). O fator de correção foi então obtido pela divisão da área foliar real pela área foliar calculada de acordo com Barros et al. (1973). Foram efetuadas análises de variância para verificar se as características de comprimento, largura, área foliar real, área foliar calculada e fator de correção foram afetadas pelas diferenças de amostras entre os terços inferior, médio e superior do dossel das plantas.

A área foliar foi também estimada por meio de regressões, testando-se os modelos: linear e não linear (quadrático, cúbico e potencial), utilizando o comprimento, a largura e o produto do comprimento pela largura como variável independente, e a área foliar real (medida pelo Area Meter), como variável dependente (Tabela 1).

Para gerar os modelos empíricos, foram utilizadas oito plantas, totalizando 720 folhas amostradas, e para o teste dos modelos foram utilizadas as outras três plantas, totalizando 450 folhas amostradas. A 
escolha do melhor modelo foi realizada por métodos estatísticos, utilizando-se como critério do coeficiente de determinação $\left(\mathrm{R}^{2}\right)$ maior ou igual a 0,90 . Para a avaliação do desempenho dos modelos entre valores estimados e observados para a estimativa da área foliar, os indicadores utilizados foram a raiz quadrada do erro quadrático médio (RMSE) e o índice de con-

$$
R M S E=\sqrt{\left(N^{-1} \cdot \sum_{i=1}^{N}\left(P_{i}-O_{i}\right)^{2}\right)}
$$

cordância $(d)$ desenvolvido por Wilmott et al. (1985), obtidos pelas equações 1 e 2 , respectivamente:

em que $P_{i}$ é o valor estimado; $O_{i}$ é o valor

$$
d=1-\frac{\sum_{i=1}^{n}(P i-O i)^{2}}{\sum_{i=1}^{n}(P i-\bar{O}|+| O i-\bar{O} \mid)^{2}}
$$

observado, e $N$ é o número de observações igual a 450 folhas;

em que $P_{i}$ é o valor estimado; $O_{i}$ é o valor observado, e $O$ é o valor médio observado. A exatidão $(d)$ está relacionada ao afastamento dos valores estimados em relação aos observados. Esse indicador apresenta valores que variam entre zero (nenhuma exatidão) e um (exatidão perfeit a). A ordenação dos dados foi realizada com auxílio de planilhas Excel, sendo calculados médias, mínimos, máximos, desvios-padrão e coeficientes de variação.

\section{RESULTADOS E DISCUSSÃO}

Na Tabela 2, são apresentadas a análise de variância do comprimento, largura, área foliar real, área foliar calculada e fator de correção entre os terços inferior, médio e superior, além dos valores médios, mínimos e máximos de cada variável. As análises de variância demonstraram que os terços não afetaram nenhuma das características estudadas, indicando que as folhas podem ser selecionadas de qualquer parte do dossel para a estimativa da área foliar.

A área foliar real variou de 16,72 a 182,60 $\mathrm{cm}^{2}$, com média de $77,98 \mathrm{~cm}^{2}$. O fator de correção igual a 0,71 obtido neste trabalho diferiu daqueles encontrados por Tyagi e Devi (1988), Prasada et al. (1994) e Araújo et al. (2005), que encontraram para a cultura da mangueira valores de 0,$86 ; 0,64 \mathrm{e}$ 0,74, respectivamente. Essas diferenças se devem, provavelmente, ao uso de um fator geral para folhas elipsoides que não é característica da cultura da mangueira, conforme observado por Tyagi e Devi (1988), bem como, às diferenças entre cultivares, condições de manejo e locais de cultivo quanto aos trabalhos desenvolvidos por Prasada et al. (1994) e Araújo et al. (2005).

Foram obtidos 12 modelos que estimaram a área foliar em função do comprimento (C), da largura (L) e do produto (CxL) (Figura 1). Todos os modelos apresentaram valor significativo $(P<0,0001)$ para estimar a AF em função das medidas lineares, demonstrando que existe correlação estreita entre a área foliar e as medidas lineares das folhas. $\mathrm{O}$ valor de $\mathrm{R}^{2}$ variou de 0,89 a 0,97 para os modelos do tipo linear (Figuras 1a, 1b e 1c) e quadrático (Figuras $1 \mathrm{~d}, 1$ e e $1 \mathrm{f})$, de 0,90 a 0,97 para o modelo do tipo cúbico ( $1 \mathrm{~g}, 1 \mathrm{~h}$ e $1 \mathrm{i})$ e de 0,90 a 0,96 para o modelo do tipo potência $(1 \mathrm{j}, 1 \mathrm{k}$ e 11$)$, sendo que os menores valores corresponderam aos modelos que utilizaram a largura máxima das folhas como variável independente. Dentre os modelos, apenas um não apresentou coeficiente de determinação $\left(\mathrm{R}^{2}\right)$ maior que 0,90 (Figura 1b).

Os modelos que utilizaram o produto $(\mathrm{CxL})$ como variável independente (Figuras 1c, 1f, 1i e 11) apresentaram valores de coeficientes de determinação $\left(\mathrm{R}^{2}\right)$ superiores aos modelos que utilizaram apenas uma das dimensões, indicando que a estimativa da área de folhas de mangueira pelo produto das dimensões lineares é a mais adequada em relação às variáveis utilizadas isoladamente. Esse resultado pode ser justificado devido á mangueira ser uma espécie que não apresenta polimorfismo foliar em diferentes idades das folhas e fases de desenvolvimento da planta, sendo suas folhas apresentadas de formato lanceolado durante todo o ciclo da cultura (CUNHA; CASTRO NETO, 2000; CUNHA et al., 2002).

Resultados semelhantes foram observados por Araújo et al. (2005) em estudos realizados com as cultivares de mangueira Tommy Atkins e Haden. Ao estimarem a área foliar pela regressão linear utilizando o comprimento, a largura e o produto do comprimento pela largura, também encontraram que as estimativas em que se utilizou o produto do comprimento pela largura apresentaram maior precisão, com valor de $\mathrm{R}^{2}$ igual a 0,99 para a cv. Haden e 0,93 para a cv. Tommy Atkins, e encontraram menor precisão quando utilizaram apenas uma das medidas lineares.

A obtenção de maior precisão com o uso de equações com mais de uma medida linear, como no caso do produto (comprimento $\mathrm{x}$ largura) foi verifica- 
da em diversas espécies por vários autores. Silva et al. (2002) concluíram que a estimativa da área foliar de plantas de gergelim são mais precisas quando se usam ambas as dimensões, comprimento e largura do limbo. Lima et al. (2008), trabalhando com a cultura do feijão-caupi, verificaram que as equações que envolveram duas medidas biométricas, a soma e o produto das dimensões, apresentaram melhor ajuste. Maracajá et al. (2008) optaram por estimar $\mathrm{a}$ área foliar de juazeiro pela equação do tipo linear que utilizou o produto $\mathrm{C} \times \mathrm{L}$, por ter apresentado menor soma de quadrados do resíduo (SQR) e valor de $\mathrm{R}^{2}$ igual a 0,9937 . Aquino et al. (2011) observaram que os modelos gerados para a estimativa da área do limbo foliar de plantas de girassol, a partir do produto $(\mathrm{CxL})$, possibilitaram o melhor ajuste com maior $\mathrm{R}^{2}$ e menor raiz do quadrado médio do erro (RQME) para as duas cultivares testadas e na análise com os dados agrupados.

No processo de validação dos modelos, as análises de regressão da área foliar com o comprimento e a largura das folhas, realizadas separadamente, apresentaram menores graus de correlação, com valores de $\mathrm{R}^{2}$ menores que 0,90 (resultados não apresentados) do que aquelas realizadas com o produto das dimensões. Os modelos que utilizaram o produto como variável independente apresentaram valores de coeficientes de determinação $\left(\mathrm{R}^{2}\right)$ acima de $95 \%$, indicando que estes conseguem explicar com maior precisão um percentual significativo da variação dos dados (Figura 2).

No desempenho dos modelos entre valores estimados e observados para a estimativa da área foliar, verificou-se que todos os modelos (linear e não linear) podem ser uma alternativa simples de estimativa da área foliar em função do produto das dimensões da folha (Tabela 3).

Queiroga et al. (2003) testaram modelos do tipo linear e não-linear para a estimativa da área foliar de feijão-vagem utilizando apenas a largura máxima dos folíolos e obtiveram melhor correlação nos modelos do tipo cúbico e potencial, com este último apresentando o menor coeficiente de variação, sendo, portanto, o mais preciso. Resultados semelhantes também foram encontrados por Lima et al. (2008) ao avaliarem equações do tipo potencial e linear para a estimativa da área foliar de feijão-caupi observando que ambos os modelos apresentaram boa precisão, sendo a equação do tipo potencial com a utilização do produto das dimensões a que apresentou melhor ajuste. Aquino et al. (2011), ao ajustarem equações do tipo linear, quadrático, cúbico, potencial e exponencial para estimativa da área foliar de girassol, observaram que os modelos do tipo potencial com variável independente $\mathrm{L}$ ou $\mathrm{CxL}$ foram os mais precisos com maior valor de $\mathrm{R}^{2}$ e menor RQME.

De modo geral, apesar de todos os modelos que estimam a área foliar em função do produto das dimensões terem alcançado valores de $\mathrm{R}^{2}$ maior que 0,95 , tanto na geração como no desempenho do modelo, o melhor resultado foi obtido com o modelo não linear cúbico, pois apresentou menor coeficiente linear, coeficiente angular do teste mais próximo de um, menor valor de RMSE e índice de concordância próximo de um, indicando perfeita concordância, ou seja, grande exatidão entre valores observados e simulados de área foliar, mostrando que existe um erro mínimo entre eles. Desta maneira, este modelo mostrou maior precisão para estimar a área foliar das plantas de mangueira.

TABELA 1 - Modelos de regressão linear e não linear testados para obtenção de equações para estimativa da área foliar de mangueiras (AF) a partir de valores de comprimento (C), largura (L) e do produto $(\mathrm{CL})$.

\begin{tabular}{clllc}
\hline Modelos* & \multicolumn{1}{c}{ Linear } & \multicolumn{1}{c}{ Quadrático } & \multicolumn{1}{c}{ Cúbico } & Potencial \\
\hline Comprimento $A F=a+b C$ & $A F=a+b_{1} C+b_{2} C^{2}$ & $A F=a+b_{1} C+b_{2} C^{2}+b_{3} C^{3}$ & $A F=a C^{b}$ \\
Largura & $A F=a+b L$ & $A F=a+b_{1} L+b_{2} L^{2}$ & $A F=a+b_{1} L+b_{2} L^{2}+b_{3} L^{3}$ & $A F=a L^{b}$ \\
Produto & $A F=a+b(C L)$ & $A F=a+b_{I}(C L)+b_{2}(C L)^{2}$ & $A F=a+b_{I}(C L)+b_{2}(C L)^{2}+b_{3}(C L)^{3}$ & $A F=a(C L)^{b}$ \\
\hline
\end{tabular}

* $C$-Comprimento; $L$ - Largura; $A F$ - Área foliar; $a$ e $b$ - parâmetros a serem estimados. 

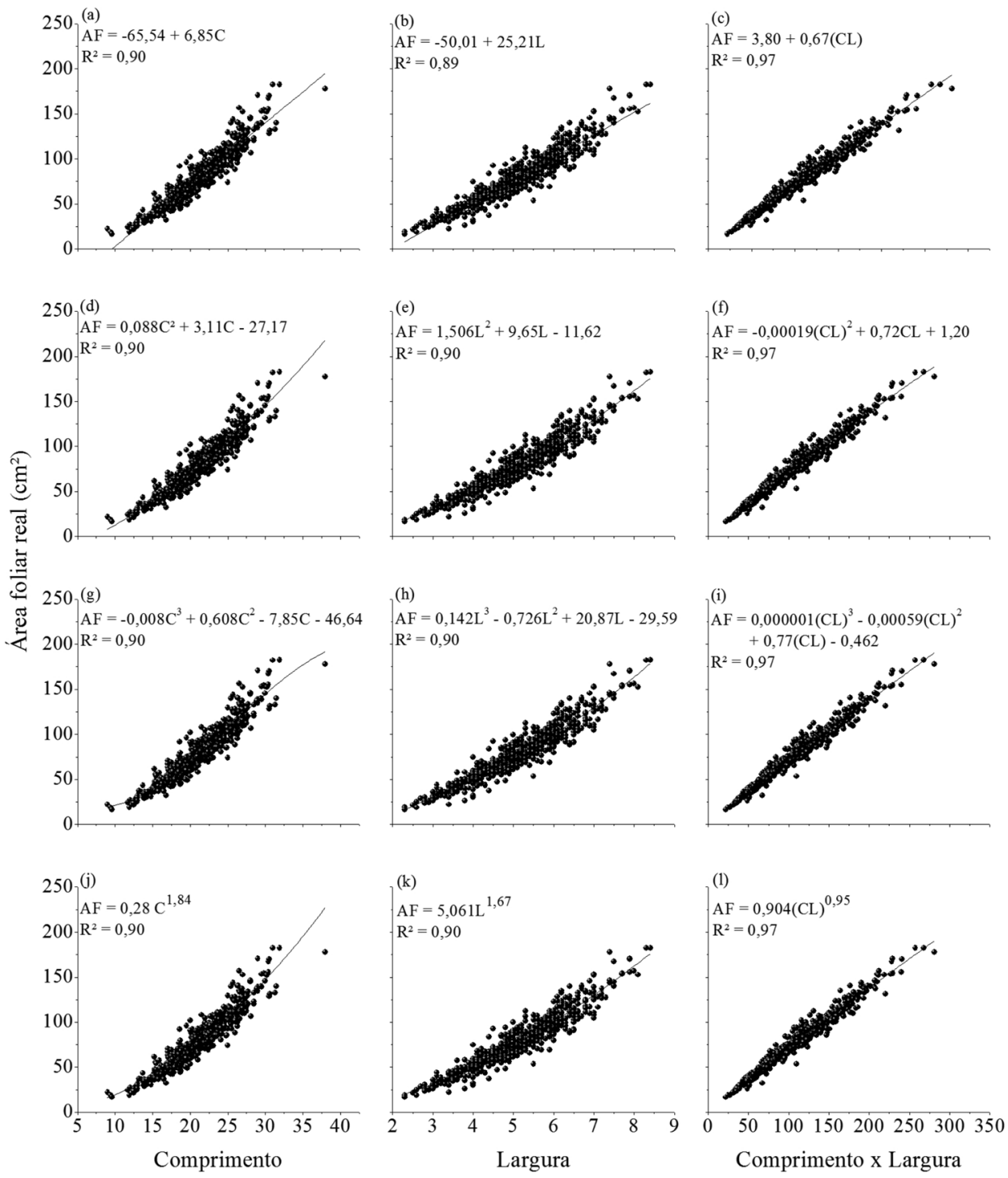

FIGURA 1 - Regressão linear (a, b, c) e não linear (d, e, f - quadrática; g, h, i - cúbica; j, k, 1 - potencial) entre a área foliar real $\left(\mathrm{cm}^{2}\right)$ e o comprimento $(\mathrm{cm})$, a largura $(\mathrm{cm})$ e o comprimento $\mathrm{x}$ a largura $\left(\mathrm{cm}^{2}\right)$ de mangueiras, cv. Tommy Atkins.
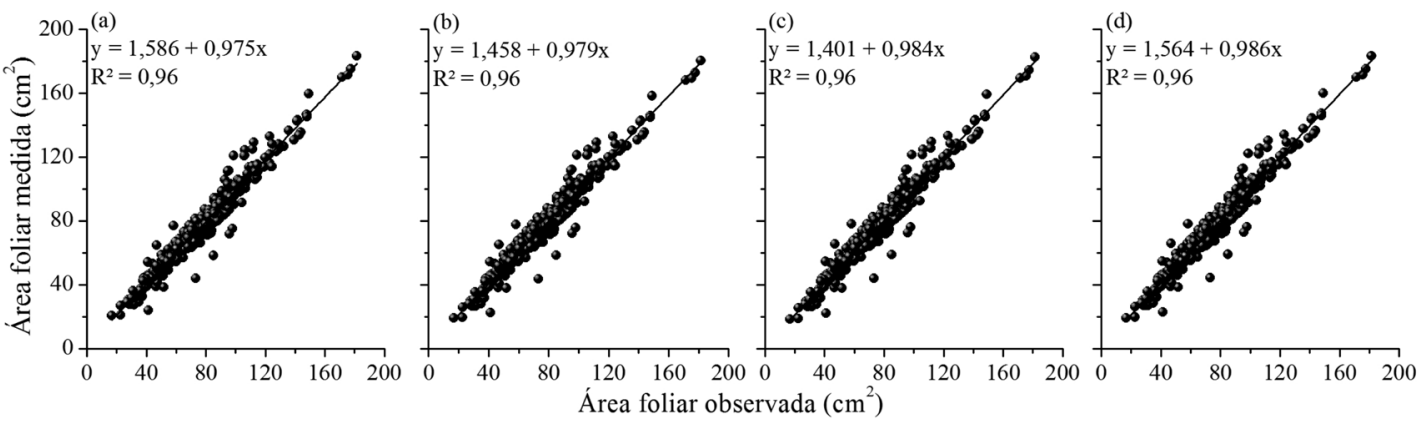

FIGURA 2 -Gráficos de correlação entre os valores de área foliar estimados pelos modelos (a-linear, b-quadrático, c-cúbico e d-potencial) e os observados pelo "Area Meter" de mangueiras, cv. Tommy Atkins. 
TABELA 2 - Comparação entre os terços inferior, médio e superior e valores médios ( \pm desvio-padrão), máximos e mínimos do comprimento $(\mathrm{C})$, largura máxima (L), área foliar real (Afr), área foliar calculada $(\mathrm{Afc}=\mathrm{CxL})$ e fator de correção $(\mathrm{f}=\mathrm{Afr} / \mathrm{Afc})$ para folhas de mangueira, $\mathrm{cv}$. Tommy Atkins.

\begin{tabular}{|c|c|c|c|c|c|}
\hline Terço & $\mathrm{C}(\mathrm{cm})$ & L (cm) & $\operatorname{Afr}\left(\mathrm{cm}^{2}\right)$ & Afc $\left(\mathrm{cm}^{2}\right)$ & f \\
\hline Inferior & $20,99^{\text {ns }} \pm 1,64$ & $5,00^{\mathrm{ns}} \pm 0,36$ & $77,17^{\mathrm{ns}} \pm 9,22$ & $107,95^{\text {ns }} \pm 15,48$ & $0,72^{\mathrm{ns}} \pm 0,02$ \\
\hline Médio & $21,38 \pm 2,20$ & $5,23 \pm 0,60$ & $81,55 \pm 15,72$ & $115,93 \pm 23,23$ & $0,71 \pm 0,02$ \\
\hline Superior & $20,63 \pm 2,59$ & $5,00 \pm 0,67$ & $75,20 \pm 18,58$ & $107,00 \pm 25,95$ & $0,71 \pm 0,02$ \\
\hline CV (\%) & 1,47 & 0,75 & 3,88 & 4,69 & 0,14 \\
\hline Valores & $\mathrm{C}(\mathrm{cm})$ & $\mathrm{L}(\mathrm{cm})$ & $\operatorname{Afr}\left(\mathrm{cm}^{2}\right)$ & $\operatorname{Afc}\left(\mathrm{cm}^{2}\right)$ & f \\
\hline Média $\pm \mathrm{s}$ & $21,00 \pm 4,03$ & $5,08 \pm 1,09$ & $77,98 \pm 29,18$ & $110,29 \pm 42,63$ & $0,71 \pm 0,05$ \\
\hline Máximo & 38,00 & 8,40 & 182,60 & 281,20 & 0,89 \\
\hline Mínimo & 9,00 & 2,30 & 16,72 & 22,08 & 0,48 \\
\hline CV $(\%)$ & 19,25 & 21,51 & 37,38 & 38,66 & 6,95 \\
\hline
\end{tabular}

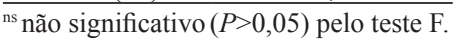

TABELA 3- Modelos de regressão, coeficientes de determinação de geração do modelo $\left(\mathrm{R}^{2}\right)$, parâmetros da equação $(a$ e $b)$, coeficiente de determinação $\left(\mathrm{R}^{2}\right)$, raiz quadrada do erro quadrático médio (RMSE) e índice de concordância $(d)$ do teste de modelos linear e não-linear, em função do comprimento $(\mathrm{C})$, da largura máxima $(\mathrm{L})$ e do produto $(\mathrm{CL})$.

\begin{tabular}{lcccccc}
\hline \multicolumn{1}{c}{ Modelo } & \multirow{2}{*}{$\mathbf{R}^{2}$} & - & & & & \\
& & $\boldsymbol{a}$ & $\boldsymbol{b}$ & $\mathbf{R}^{\mathbf{2}}$ & $\mathbf{R M S E}$ & $\boldsymbol{d}$ \\
\hline $\mathrm{AF}=3,80+0,67(\mathrm{CL})$ & 0,97 & 1,586 & 0,975 & 0,96 & 5,59 & 0,99 \\
$\mathrm{AF}=-0,00019(\mathrm{CL})^{2}+0,72(\mathrm{CL})+1,20$ & 0,97 & 1,458 & 0,979 & 0,96 & 5,58 & 0,99 \\
$\mathrm{AF}=0,000001(\mathrm{CL})^{3}-0,00059(\mathrm{CL})^{2}+0,77(\mathrm{CL})-0,462$ & 0,97 & 1,401 & 0,984 & 0,96 & 5,58 & 0,99 \\
$\mathrm{AF}=0,904(\mathrm{CL})^{0,95}$ & 0,97 & 1,564 & 0,986 & 0,96 & 5,61 & 0,99 \\
\hline
\end{tabular}

\section{CONCLUSÕES}

1-O fator de correção de 0,71 pode ser utilizado para estimar a área foliar real da mangueira, multiplicado pelas dimensões lineares.

2-Os modelos que utilizam o produto das dimensões lineares (comprimento x largura) são os mais adequados para estimar a área das folhas da mangueira, uma vez que apresentam maior correlação, sendo o modelo não linear do tipo cúbico o mais preciso.

\section{REFERÊNCIAS}

ARAÚJO, E.C.E.; SANTOS, E.P.dos; PRADO, C.H.B. de A. Estimativa da área foliar da mangueira (Mangifera indica L.) cvs. Tommy Atkins e Haden, utilizando dimensões lineares. Revista Brasileira de Fruticultura, Jaboticabal, v.27, n.2, p.308-309, 2005.
ASNER, G.P.; SCURLOCK, J.M.O.; HICKE, J.A. Global synthesis of leaf area index observations: implications for ecological and remote sensing studies. Global Ecology and Biogeography, Oxford, v.12, p.191-205, 2003.

AQUINO, L.A. de; SANTOS JÚNIOR, V.C. dos; GUERRA, J.V.S.; COSTA, M.M. Estimativa da área foliar do girassol por método não destrutivo. Bragantia, Campinas, v.70, n.4, p.832-836, 2011.

BARROS, R.S.; MAESTRI, M.; VIEIRA, J.; BRAGA FILHO, L.J. Determinação da área foliar do café (Coffea arábica L. cv. 'Bourbon Amarelo'). Revista Ceres, Viçosa, v.20, n.107, p.44-52, 1973.

CUNHA, G.A.P. de; CASTRO NETO, M.T. de. Aspectos botânicos. In: MATOS A.P. de (Org.). Manga. Produção: aspectos técnicos. Cruz das Almas: Embrapa Mandioca e Fruticultura; Brasília: Embrapa Comunicação para Transferência de Tecnologia, 2000. 63p. 
CUNHA, G.A.P. da; PINTO, A.C de Q.; FERREIRA, F.R. Origem, Dispersão, Taxonomia e Botânica. In: GENÚ, P.J. de C.; PINTO, A.C de A. (Ed.). A Cultura da mangueira. Brasília: Embrapa Informação Tecnológica, 2002. p.31-36.

FAGUNDES, J.D.; STRECK, N.A.; KRUSE, N.D. Estimativa da área foliar de Aspilia montevidensis (Spreng.) Kuntze utilizando dimensões lineares. Revista Ceres, Viçosa, MG, v.56, n.3, p.266-273, 2009.

FAVARIN, J.L.; DOURADO NETO, D.; GARCÍA Y GARCÍA, A.; VILLA NOVA, N.A.; FAVARIN, M. da G.G.V. Equações para a estimativa do índice de área foliar do cafeeiro. Pesquisa Agropecuária Brasileira, Brasília, v.37, n.6, p.769-773, 2002.

JONCKHEERE, I.; FLECK, S.; NACKAERTS, K.; MUYS, B.; COPPIN, P.; WEISS, M.; BARET, F. Review of methods for in situ leaf area index determination. Part I. Theories, sensors and hemispherical photography. Agricultural and Forest Meteorology, Amsterdam, v.121, p.19-35, 2004.

LIMA, C.J.D.S.; OLIVEIRA, F.A.; MEDEIROS, J.F.; OLIVEIRA, M.K.T.; OLIVEIRA FILHO, A.F. Modelos matemáticos para estimativa de área foliar de feijão-caupi. Revista Caatinga, Mossoró, v.21, n.1, p.120-127, 2008.

MARACAJÁ, P.B.; MADALENA, J.A.S. da; ARAÚJO, E. de; LIMA, B.G; LINHARES, P.C.F. Estimativa de Área Foliar de Juazeiro por Dimensões Lineares do Limbo Foliar. Revista Verde, Mossoró, v.3, n.4, p.0-05, 2008.

MONTEIRO, J.E.B.A.; SENTELHAS, P.C.; CHIAVEGATO, E.J.; GUISELINI, C.; SANTIAGO, A.V.; PRELA, A. Estimação da área foliar do algodoeiro por meio de dimensões e massa das folhas. Bragantia, Campinas, v.64, n.1, p.15-24, 2005.

PEREIRA, A.R.; VILLA NOVA, N.A.; SEDIYAMA, G.C. Evapo(transpi)ração. Piracicaba: FEALQ/ ESALQ/USP, 1997. p.183.
PRASADA, R.G.S.L.H.V.; SIBY, S.; RAO, G.L.S.H.V.P.; SEBASTIAN, S. Estimation of leaf area in tree crops. Journal of Plantation Crops, Kasaragod, v.22, n.1, p.44-46, 1994.

QUEIROGA, J.L.; ROMANO, E.D.U.; SOUZA, J.R.P.; MIGLIORANZA, E. Estimativa da área foliar do feijão-vagem (Phaseolus vulgaris L.) por meio da largura máxima do folíolo central. Horticultura Brasileira, Brasília, v.21, n.1, p.64-68, 2003.

ROUPHAEL, Y.; COLLA, G.; FANASCA, S.; KARAM, F. Leaf area estimation of sunflower leaves from simple linear measurements. Photosynthetica, Prague, v.45, n.2, p.306-308, 2007.

SILVA, L.C.; SANTOS, J.W.; VIEIRA, D.J.; BELTRÃO, N.E.M.; ALVES, I.; JERÔNIMO, J.F. Um método simples para se estimar área foliar de plantas de gergelim (Sesamum indicum L.). Revista Brasileira de Oleaginosas e Fibrosas, Campina Grande, v.6, n.1, p.491-496, 2002.

SINOQUET, H.; STEPHAN, J.; SONOHAT, G.; LAURI, P.É.; MONNEY, P.H. Simple equations to estimate light interception by isolated trees from canopy structure features: assessment with threedimensional digitized apple trees. New Phytologist, Cambridge, v.175, p.94-106, 2007.

TAKEDA, T.; OGUMA, H.; SANO, T.; YONE, Y.; FUJINUMA, Y. Estimating the plant area density of a Japanese larch (Larix kaempferi Sarg.) plantation using a ground-based laser scanner. Agricultural and Forest Meteorology, Amsterdam, v.148, p.428438, 2008.

TYAGI, D.N.; DEVI, K.M.T.M. Physiology of mango (Mangifera indica L.): I. Preliminary studies on the physiological characteristics of leaf. Indian Journal of Plant Physiology, New Delhi, v.31, n.4, p.368-373, 1988.

WILMOTT, C.J.; ACKLESON, S.G.; DAVIS, R.E.; FEDDEMA, J.J.; KLINK, K.M.; LEGATES, D.R.; O'DONNELL, J.; ROWE, C.M. Statistics for the evaluation and comparison of models. Journal of Geophysical Research, Ottawa, v.90, n.5, p.8995$9005,1985$. 